\title{
Antibiotic Resistance Pattern of Bacteria Isolated from Patients of Urinary Tract Infections in Iraq
}

\author{
Mohemid M. Al-Jebouri ${ }^{1}$, Salih A. Mdish ${ }^{2}$ \\ ${ }^{1}$ Deapartment of Microbiology, College of Medicine, University of Tikrit, Tikrit, Iraq \\ ${ }^{2}$ Department of Clinical Laboratory, Tikrit Teaching Hospital, Tikrit, Iraq \\ Email: profaljebouri@yahoo.com
}

Received December 28, 2012; revised January 30, 2013; accepted February 8, 2013

Copyright (C) 2013 Mohemid M. Al-Jebouri, Salih A. Mdish. This is an open access article distributed under the Creative Commons Attribution License, which permits unrestricted use, distribution, and reproduction in any medium, provided the original work is properly cited.

\begin{abstract}
Objectives: The main objective of this study was to investigate the bacterial infections of urinary tract in patients with urolithiasis. The patients were referred to and/or visiting the Urology Department in Tikrit Teaching Hospital in Tikrit city. Methods: The present study was carried out from November 2011 until July 2012. One hundred and sixty patients with urolithiasis were included in the present study whose ages ranged from 12 to 70 years. Male to female ratio was almost 2:1. The control group consisted of two groups; group one consisted of 40 persons ( 20 males and 20 females) who were selected randomly from Tikrit city during the period of this study and they had no previous personal nor family history of urolithiasis, while group two consist of 40 persons (20 males and 20 females) infected with urinary tract infection. Urine culture was done for all the patients and the control group. Results: Forty five percent (45/160) of these had UTI's. Six patients revealed mixed bacterial growth. Eighty-four percent (38/45) of patients with UTI were infected with gram negative organisms where as only $16 \%(7 / 45)$ of patients were infected with gram positive organisms. The majority of the gram negative bacteria isolated belonged to the family Enterobacteriaceae particularly E. coli as it was isolated from 14 (31\%) cases. The urease producing bacteria were isolated from 31 cases (50\%). Conclusions: UTI's in urolithiasis was more frequent in females than males and the percentages were 28/45 and 17/45 respectively. The most effective antimicrobial agent was amikacin and the least effective one was ampicillin, whereas ciprofloxacin, cefoxitin, levofloxacin, nitrofurantoin, nalidixic acid, chloramphenicol, amoxycillin and gentamicin were effective at different levels.
\end{abstract}

Keywords: Antibiotic Resistance; UTI; Pathogens; Iraq

\section{Introduction}

Antibiotic resistance is a specific type of drug resistance when a microorganism has the ability of withstanding the effects of antibiotics. Antibiotic resistance evolves via natural selection acting upon random mutation, but it can also be engineered by applying an evolutionary stress on a population. Urinary Tract Infections (UTIs) are one of the most prevalent extra-intestinal bacterial infections. Nowadays, it represents one of the most common diseases encountered in medical practice affecting people of all ages from the neonate to the geriatric age group [1]. Worldwide, about 150 million people are diagnosed with UTI each year [2]. Most infections are caused by retrograde ascent of bacteria from the faecal flora via the urethra to the bladder and kidney especially in the females who have a shorter and wider urethra and are more readily transferred by microorganisms [3]. The structure of the females urethra and vagina makes it susceptible to trauma during sexual intercourse as well as bacteria being massaged up the urethra and into the bladder during pregnancy and/or child birth [4,5]. Majority of UTIs are not life threatening and do not cause any irreversible damage. However, when the kidneys are involved, there is a risk of irreparable tissue damage with an increased risk of bacteremia [6]. The emergence of antibiotic resistance in the management of UTIs is a serious public health issue, particularly in the developing world where apart from high level of poverty, ignorance and poor hygienic practices, there is also high prevalence of fake and spurious drugs of questionable quality in circulation. Studies aimed at gaining knowledge about the type of pathogens responsible for UTIs and their susceptibility patterns may help the clinicians to choose the right em- 
pirical treatment.

\section{Materials and Methods}

\subsection{Source of Specimens}

This study was conducted in the Urology Department in Tikrit Teaching Hospital as a part of research programs for higher studies in College of Medicine, University of Tikrit. The number of the patients was 160 and they were recruited from November 2011 until July 2012. All have urolithiasis including 104 males and 54 females. Patients aged between 15 to 70 years.

The control group composed of 80 subjects who were looked healthy and had comparable criteria to the patients and composed of 40 males and 40 females.

\subsection{Laboratory Methods}

\subsubsection{Culture of Urine Specimens}

Media were prepared and sterilized according to the manufacturer's instruction. The prepared media used for isolation, determination of the viable count, identification and susceptibility testing were carried out after being solidified [7].

\subsubsection{Preparation of the Culture Medium for Antibiotic Sensitivity Testing}

Antibiotic sensitivity testing of all isolates was performed on Mueller-Hinton medium by the Kerby Bauer method (1996) following the definition of the National Committee of Clinical Laboratory Standard (NCCLS, 1999). The medium was allowed to cool at $45^{\circ} \mathrm{C}$ and poured into Petri dishes to about $4 \mathrm{~mm}$ thickness of medium. The solidified plates were incubated at $37^{\circ} \mathrm{C}$ for 15 30 minutes to let the excess moisture to evaporate (Fisher scientific, USA) [7].

\subsubsection{Inoculation and Incubation}

The plates were inoculated by dipping a sterile swab into the inoculum, the excess inoculum was removed by pressing and rotating the swab firmly against the side wall of the tube above the level of fluid, then the swab was rubbed all over the surface of the medium, rotating the plate 3 times at an angle of 60 degree after each application and finally the swab passed around the edge of agar surface. The plate was left to dry at room temperature with the lid closed for few minutes. After 15 minutes of inoculation, the antibiotic discs were applied and the plates were inverted for incubation to avoid accumulation of moisture on the agar surface [8]. Maximum 5 antibiotic discs were selected and placed onto each plate using flamed forceps for application of the discs on the plate and each disc pressed down gently to ensure even contact with the medium. After overnight incubation at $37^{\circ} \mathrm{C}$ the diameter of each zone including the diameter of zone inhibition was measured and recorded in $\mathrm{mm}$ and compared with the standard inhibition zone. For motile organisms, e.g. Proteus spp. the swarming haze was ignored and zones were measured at the point where growth was obviously inhibited [8].

\subsubsection{Statistical Analysis}

Statistical analyses in the present study were done by using Microsoft Office Excel 2007, SPSS version 12 (Statistical Package for Social Sciences). The programs used were F-test, T-test, least significant difference (LSD) and Chi-square [9].

\section{Results}

\subsection{Urine Culture Result for the Patients}

Urine culture had been done for all the 160 patients who were included in this study. Only 45 patients had positive urine culture as shown in Figure 1. Twenty-eight of them were females and 17 were males. The most common organism was $E$. coli which was isolated from 14 patients with percentage of $31.1 \%$. E. coli was the most prevalent followed by Proteus mirabilis, Pseudomonas aeruginosa, Staphylococcus aureus, Klebsiella pneumoniae, Staphylococcus Saprophyticus and Serratia marcescenes and the frequencies of isolation were $22.2 \%(10)$, $15.5 \%$ (7), 11.1\% (5), 7.4\% (5), 13.3\% (6), 4.4\% (2) and $2.2 \%$ (1) respectively. Statistically, there was highly significant difference between males and females according to the distribution of the isolated bacteria ( $\mathrm{P}$ value $\leq$ $0.05)$.

\subsection{Antimicrobial Sensitivity Testing}

The antibiotic sensitivity test was carried out using disc diffusion technique for all the bacterial isolates to the most commonly antibiotic agents that used in the treatment of UTI as it was shown in Figures 2-12. It was found that different bacterial pathogens were highly sensitive to amikacin, ciprofloxacin and most of them were also highly sensitive to nitrofurantoin, nalidixic acid and cefotaxin. The present study also shows that the most resistant bacteria were Pseudomonas aeruginosa and almost $80 \%$ of its isolates were still sensitive to amikacin and ciprofloxacin. Almost all isolates (100\%) of EScherichia coli were highly sensitive to levofluxacin, cefoxitin and chloromphenicol. E. coli isolates were resistant to nalidixic acid and almost $3 \%$ only of them were sensitive to nalidixic acid. Most of the pathogens isolated were moderately sensitive to gentamicin. Staph. aureus isolates were highly resistant to ampicillin and amoxycillin (Figures 2 and 3). The overall assessment of the most antibiotics used for UTI treatment revealed that amikacin 


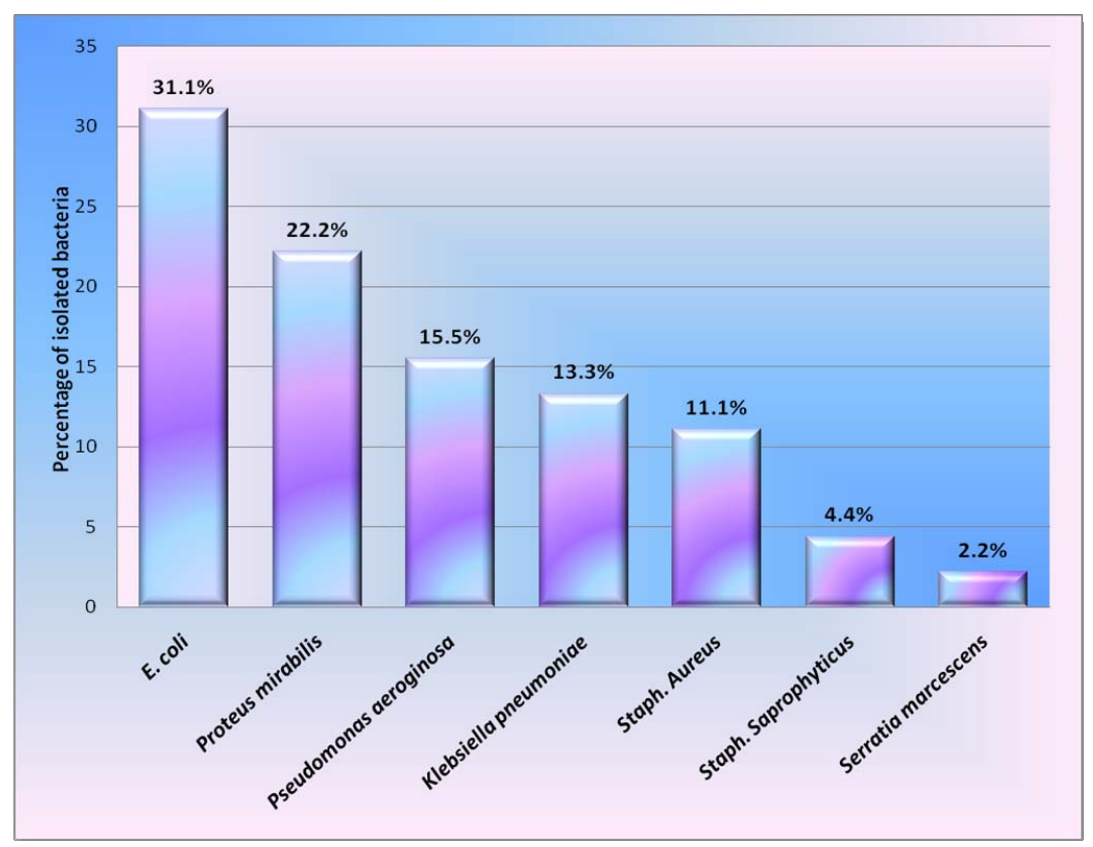

Figure 1. Percentage of isolated bacteria from urine culture of the patients.

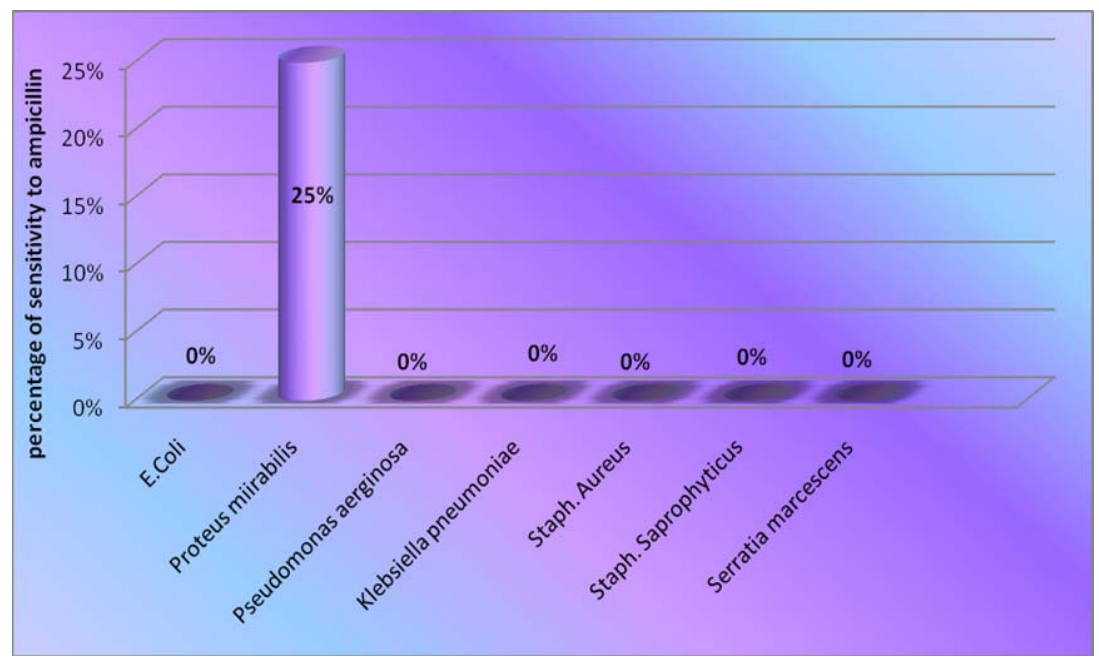

Figure 2. Sensitivity of isolated strains to ampicillin.

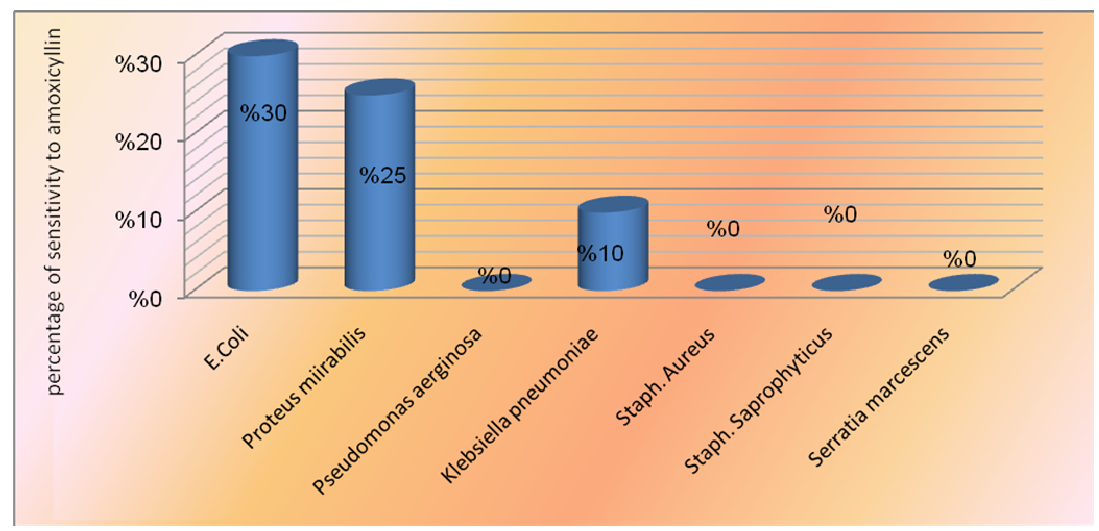

Figure 3. Sensitivity of isolated strains to amoxycillin. 


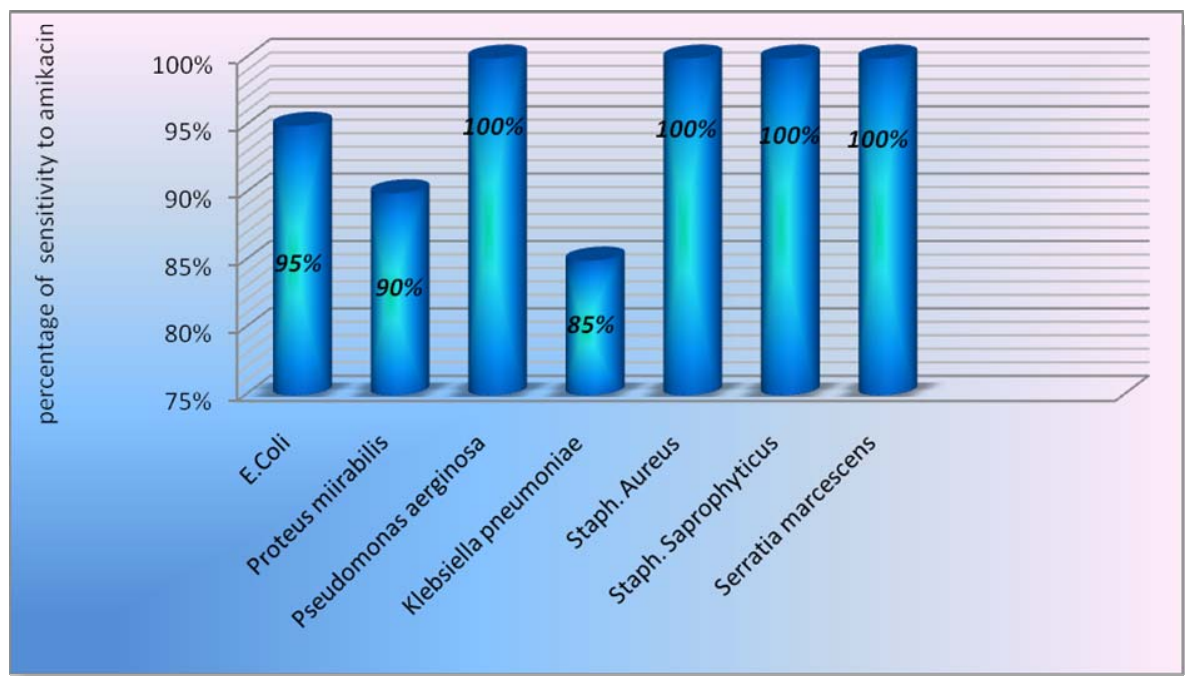

Figure 4. Sensitivity of isolated strains to amikacin.

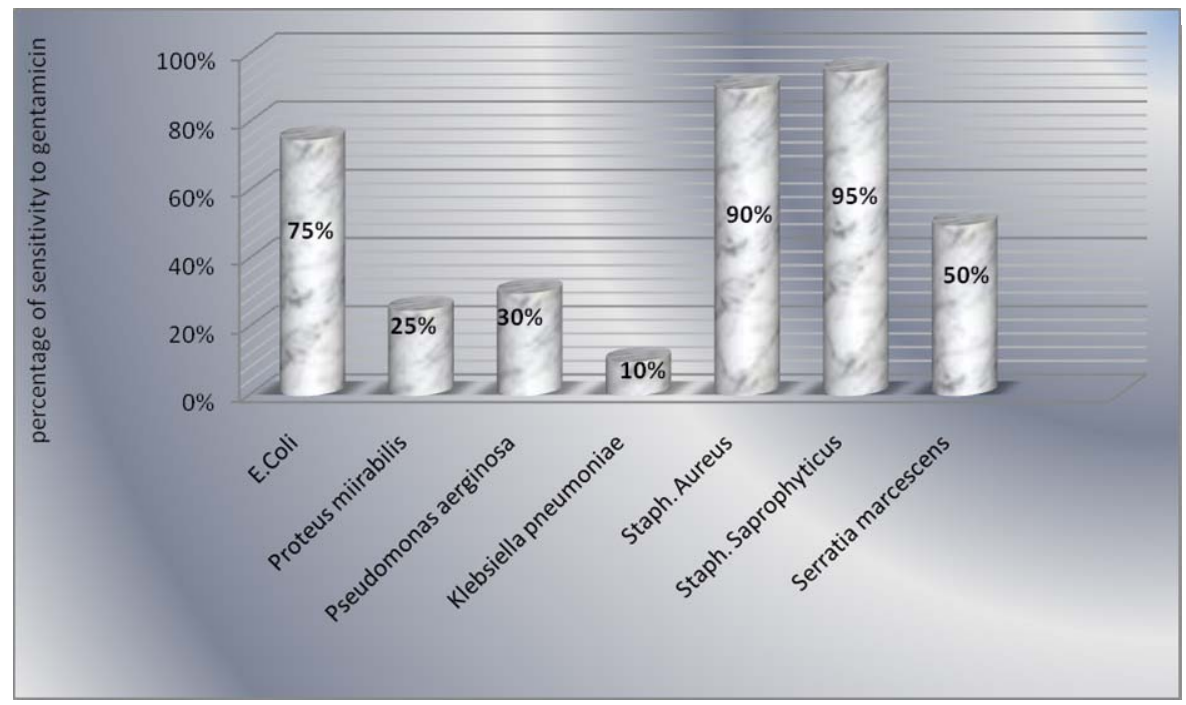

Figure 5. Sensitivity of isolated strains to gentamicin.

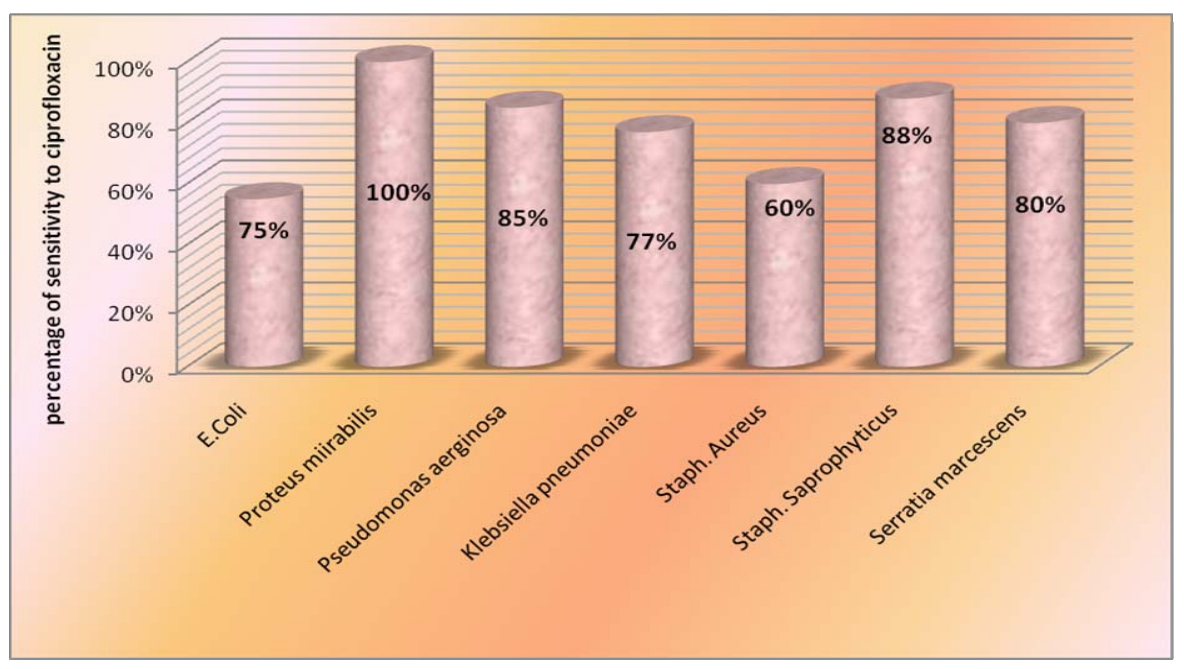

Figure 6. Sensitivity of isolated strains to ciprofloxacin. 


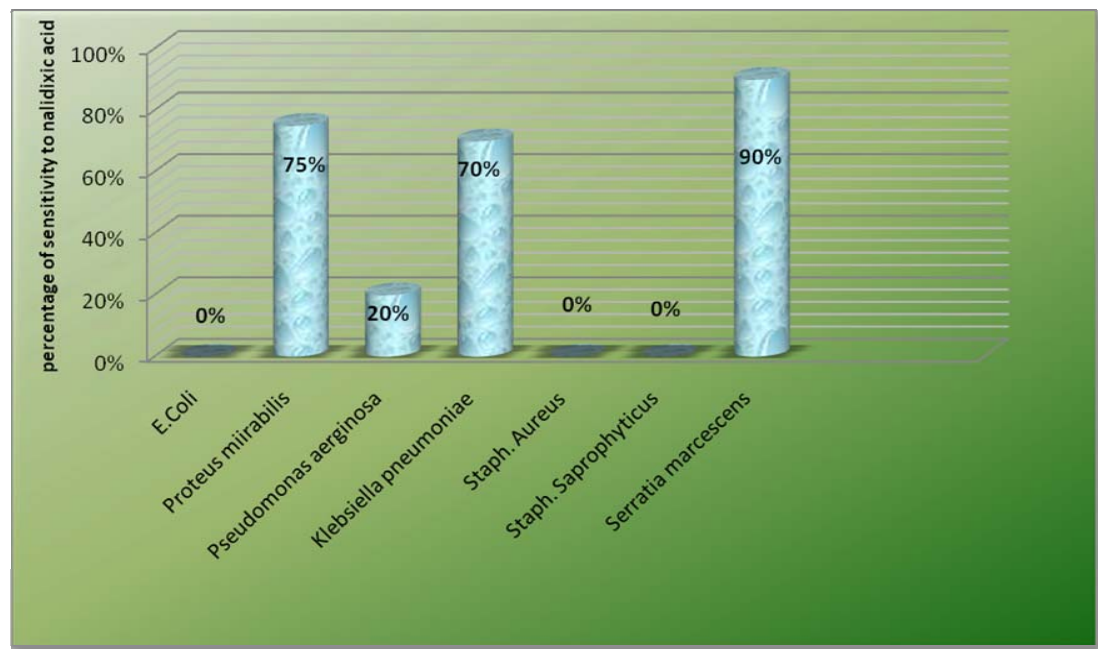

Figure 7. Sensitivity of isolated strains to nalidixic acid.

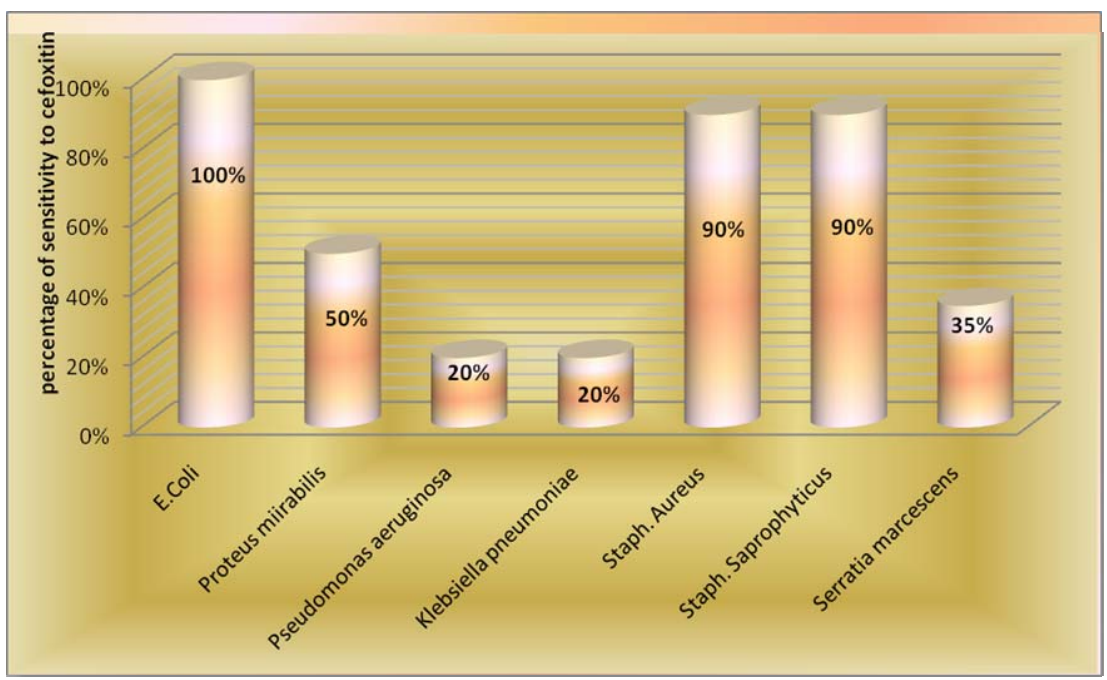

Figure 8. Sensitivity of isolated strains to cefoxitin.

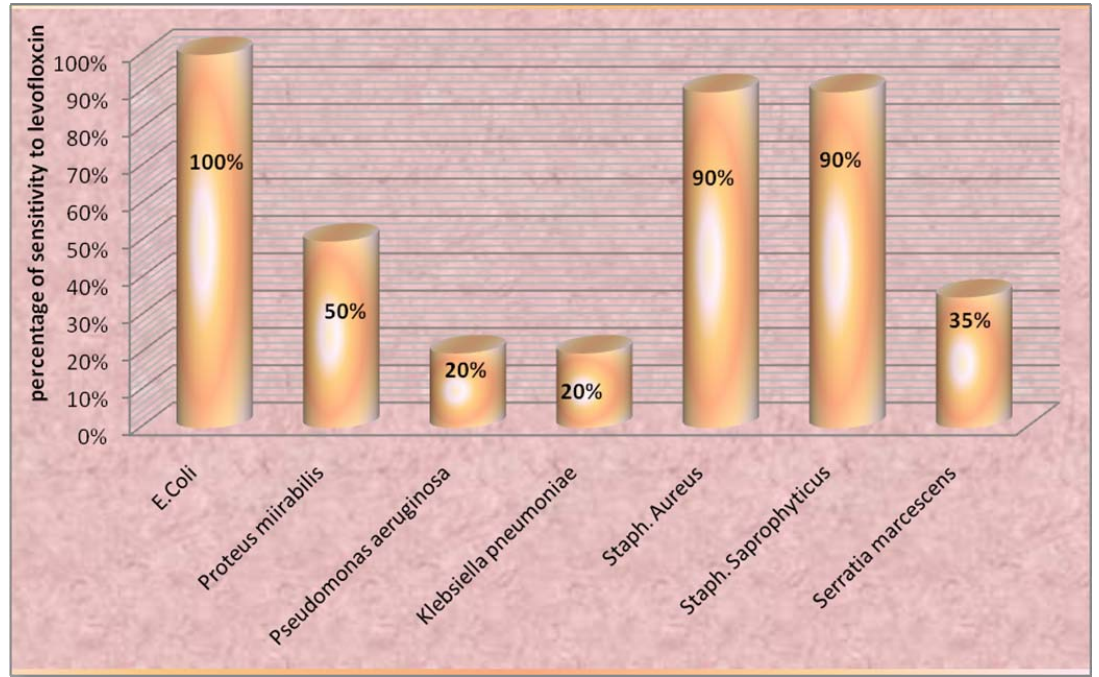

Figure 9. Sensitivity of isolated strains to levofloxacin. 


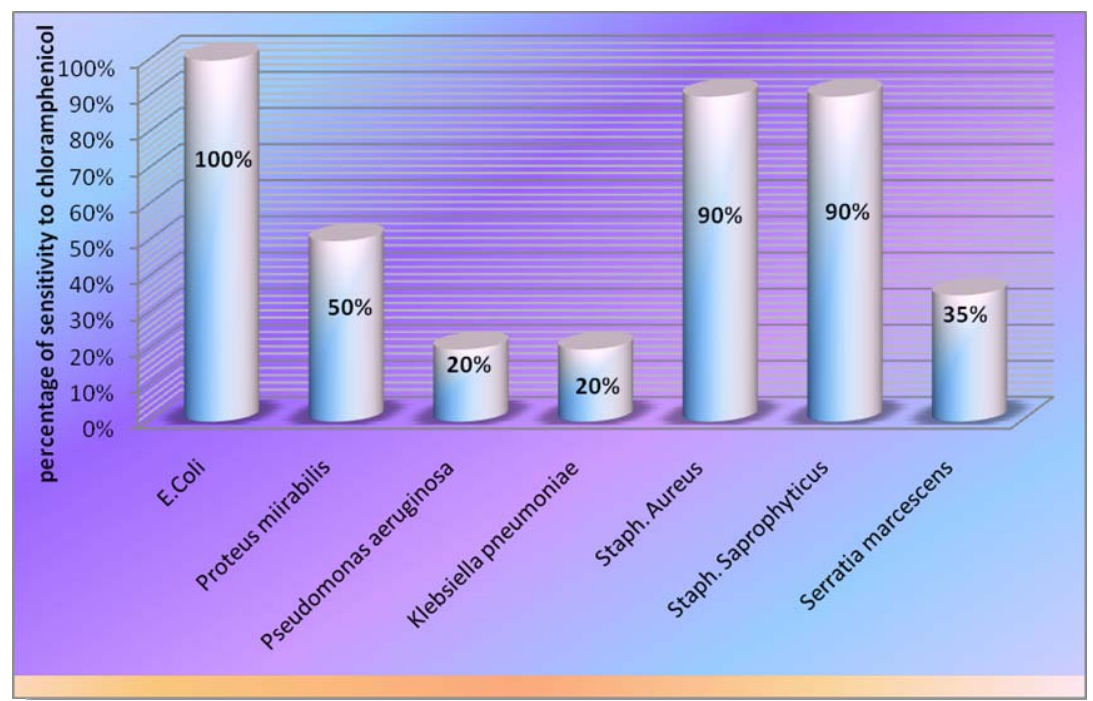

Figure 10. Sensitivity of isolated strains to chloramphenicol.

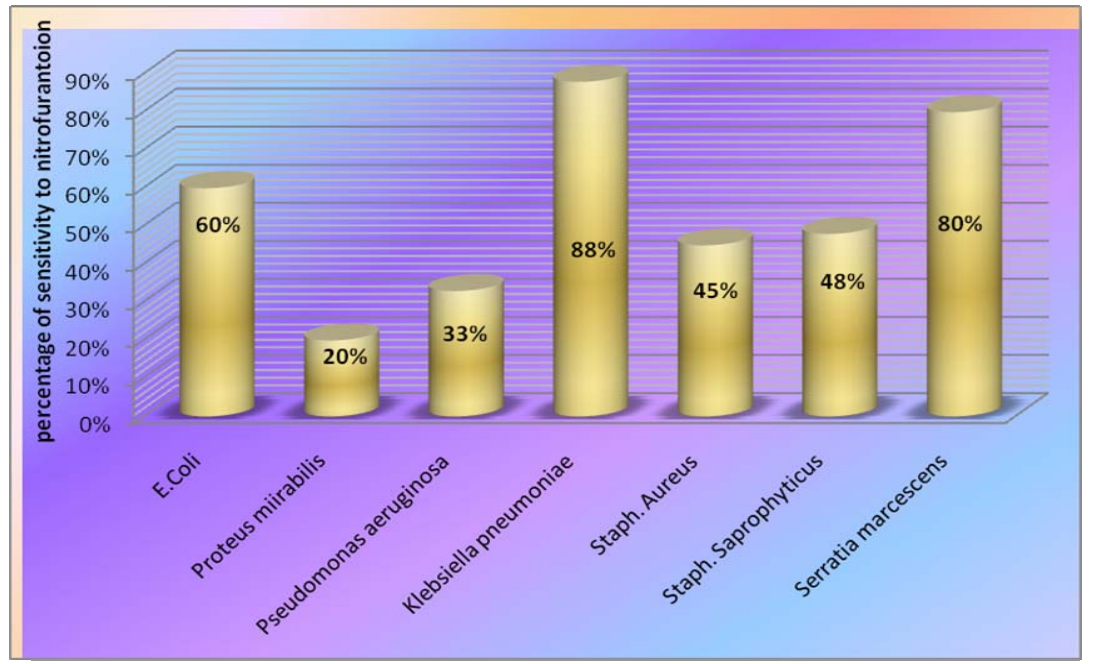

Figure 11. Sensitivity of isolated strains to nitrofurantoin.

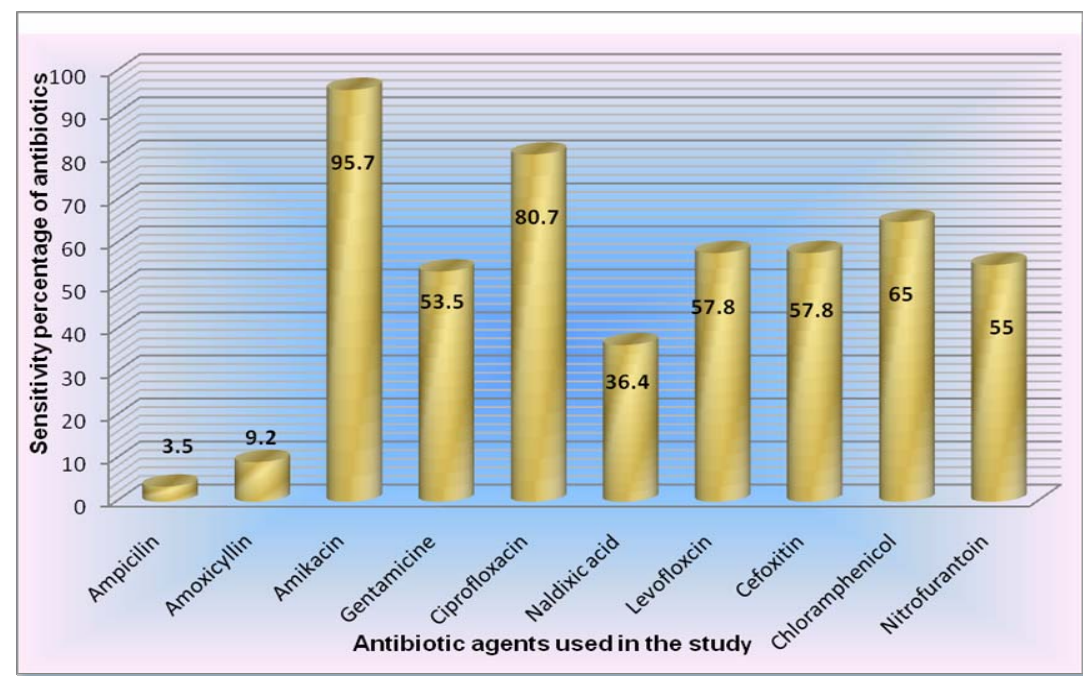

Figure 12. Total sensitivity testing for overall antibiotics used in this study. 
was the most effective and almost all the isolates were resistant to ampicillin (Figure 12). Almost $50 \%$ of the isolates of different pathogens were sensitive to nitrofurantoin (Figure 12).

\section{Discussion}

Urine culture for all 160 patients was made, which was positive for 45 (28\%) patients as shown in Figure 1. This was almost similar to that found elsewhere $l[9,10]$. In contrast, the present results were almost different from those reported by Al-Jebouri [11] and Al-Jebouri and hasen [12]. However, E. coli was the most prevalent organisms causing UTI among patients studied and this conclusion was found elsewhere [13]. E. coli as a commonest cause of UTI may be due to certain virulence factors like hemolysin production and presence of fimbriae. Moreover, Serratia marcescens was rarely isolated $2.2 \%$ from the UTI cases inviestigated here and almost the same pattern of isolation was reported by Manikandan et al. in India [13]. The present study revealed other organisms to be associated with Iraq patients who suffered from UTI and these organisms were reported previously by Al-Jebourians Hasen [12]. The most organisms caused UTI in this study were belonging to gram negative bacteria which were isolated from 38 (84.4\%) patients. These results were almost similar to those of Al-Jebouri (11), Al-Rawi [14] and Navaneeth et al. [15]. It was shown in Figure 8 that $7(4.3 \%)$ of the patients with infection stones were having UTI while only 38 (24\%) of patients with noninfection stone were having UTI as was proven by urine culture. Statistically, there was no significant difference in distribution of positive urine culture between infection and noninfection stones ( $\mathrm{P}$ value $>0.1)$. These results were almost comparable with those found by Al-Jebouri [11]. The prevalence of UTI was higher among females than male patients (in a ratio of 1.6:1) and this was almost similar to that of Al-Nass et al. [16]. Women are more prone to have UTI than men because in females, the urethra is much shorter and closer to the anus than in males, and they lack the bacteriostatic properties of prostatic secretions [17].

The overall percentages of resistance of all isolates to the antimicrobial agents were 66.5 to ampicillin, 90.8 to amoxycillin, 4.3 to amikacin, 46.5 to gentamicin, 19.3 to ciprofloxacin, 63.6 to nalidixic acid, 42.8 levofloxacin, 42.2 to cefoxitin, 35 to cloromphenicol and 45 to nitrofurantoin (Figure 12). The present study showed a high elevation in antibiotic resistance of pathogens isolated fron the Iraqi patients compared to previous years [18]. This might be due to misuse of antibiotics, usage of antibiotics from unknown origin, i.e. from uncontrol source of production, utilizing of inactivated antimicrobials, selective pressure of antibiotics and lacking of quality control on some sources of antibiotics entering Iraq es- pecially by the private sector. However, the present study showed that almost all types of pathogens causing UTI were resistant to ampicillin and most of them were highly resistant to amoxycillin. Moreover, the study conducted by Manikandan et al. [13] revealed that almost $60 \%$ of the pathogens causing UTI were resistant to amoxycillin. In contrast, Akortha and Ibadin found that most of their isolates were sensitive to nalidixic acid [19]. However, a significant increase in resistance of pathogenic strains to SXT, ampicillin and cephalothin has been found worldwide [20] but certain agents like gentamicin and nitrofurantoin still show a moderate efficacy against UTI pathogens because of its multiple mechanisms of action seem to have enabled it to retain potent activity against pathogens [21]. Furthermore, E. coli, Proteus mirabilis, Ps. aeruginosa, $S$. aureus, $K$. pneumoniae and $S$. marcesens were still highly sensitive to amikacin, ciprofloxacin and chloromphenicol (Figure 12). However, chloromphenicol is not preferred to be commonly used for medication as it might because ablastic anemia. Furthermore, the most common UTI pathogens and highly resistant to antibiotics emphasize the need for judicious use of antibiotics.

\section{Conclusion}

The susceptibility and resistance profile of all isolates in the current study have shown that ampicillin and amoxycillin possess the lower efficacy while amikacin and ciprofloxacin possess the higher efficacy. There was a general increase in the resistance pattern of isolates to all the antibiotics used in this study. The current findings confirm that bacterial resistance would be the greatest and frightening problem in our country. A new generation of antibiotics is still locally working in a higher rate. The usage of antibiotics in our locality of the world needs to be under health assessment and control with more laboratory investigations to explore the intrinsic and extrinsic parameters led to conclude high rate of resistance occurred in the local pathogens that might be disseminated to other geographical areas.

\section{REFERENCES}

[1] M. M. A. Jebouri and N. Atala, "A Study on the Interrelationship between Renal Calculi, Hormonal Abnormalities and Urinary Tract Infections in Iraqi Patients," Open Journal of Urology, Vol. 2, No. 1, 2012, pp. 6-10. doi:10.4236/oju.2012.21002

[2] K. A. Gupta, "Increasing Antibiomicrobial Resistance and the Management of Uncomplicated CommunityAcquired Urinary Tract Infections," International Journal of Antimicrobial Agents, Vol. 135, No. 2, 2001, pp. 41-50.

[3] R. A. Jones, H. I. Inabo and H. B. I. Obanibi, "Antimicrobial Suseptibilityof Some Urinary Tract Clinical Isolatesto Commonly Used Antibiotics," African Journal of 
Biotechnology, Vol. 21, No. 2, 2006, pp. 973-978.

[4] N. W. El-Sweih, W. Jamal and V. O. Rotimi, "Spectrum and Antibiotic Resistance of Uropathogens Isolated from Hospital and Community Patients with Urinary Tract Infections in Two Large Hospitals in Kuwait," Medical Principles and Practice, Vol. 14, No. 6, 2008, pp. 401407. doi: $10.1159 / 000088113$

[5] A. S. Kolawale, O. M. Kolawale, Y. T. Kandaki-Olukemi, et al., "Prevalence of Urinary Tract Infections among Patients Attending Dalhatu Araf Specialist Hospital, Lafia, Nasarawa State, Nigeria," International Journal of Medical Sciences, Vol. 1, No. 5, 2009, pp. 163-167.

[6] H. Hvidberg, C. Struve, K. A. Krogfelt, et al., "Development of a Long-Term Ascending Urinary Tract Infection Mouse Model for Antibiotic Treatment Studies," Antimicrobial Agents and Chemotherapy, Vol. 44, No. 1, 2000, pp. 156-163. doi:10.1128/AAC.44.1.156-163.2000

[7] P. W. Baker, P. Coyle, R. Bais, et al., "Influence of Season, Age and Sex on Renal Stone Formation in South Australia," Medical Journal of Australia, Vol. 156, No. 4, 1993, pp. 390-392.

[8] B. A. Forbes, D. F. Sahm, A. S. Weissfeld, et al., "Baily and Scott Diagnostic Microbiology," 12th Edition, Mosby Elsevier, Philadeliphia, 2007, pp. 93-107,187-197,842-854.

[9] J. W. Sutherland, J. H. Parks and F. L. Coe, "Recurrence after a Single Renal Stone in a Community Practice," Miner Electr1985, Metab11, 1985, pp. 267-269.

[10] H. Alpay, A. O. GokceI and N. Biyikli, "Clinical and Metabolic Features of Urolithiasis and Microlithiasis in Children," Pediatric Nephrolology, Vol. 24, No. 11, 2009, pp. 2203-2209. doi:10.1007/s00467-009-1231-9

[11] O. A. H. Al-Jebouri, "The Relationship between Urinary Caliculi Types and Urinary Tract Infections among Patients in Tikrit District," M. Sc. Thesis, College of Medicine, Tikrit University, Tikrit, 2006.

[12] M. M. Al-Jebouri and A. H. Hasen, "Vitamin $D_{3}$ Variation between Children and Adults with Reference to Re- nal Stones, Environment and Urinary Tract Infections," Open Journal of Urolology, Vol. 2, No. 3, 2012, pp. 119126.

[13] S. Manikandan, S. Ganesapandian, M. Singh, et al., "Antimicrobial Susceptibility Pattern of Urinary Tract Infection Causing Human Pathogenic Bacteria," Asian Journal of Medical Sciences, Vol. 3, No. 2, 2011, pp. 56-60.

[14] S. K. Al-Rawi, "UTIs in Diabetic Pregnant Women," M. Sc. Thesis, College of Medicine/Al-Mustansiriya University, Baghdad, 1998.

[15] T. Al-Naas, S. Al-Uqaily and B. Othman, "Urinary Calculi: Bacteriological and Chemical Association," Easter Mediterranean.

[16] J. S. AL-Bedri, "Urinary Tract Infections in the Medical City Teaching Hospital," Diploma Dissertation, Collage of Medicine/Baghdad University, Baghdad, 1987.

[17] M. M. Al-Jebouri, "The Effect of Sublethal Concentrations of Disinfectants on Antibiotic-Resistant Staphylococcus aureus," Journal of Hospital Infection, Vol. 14, No. 4, 1989, pp. 14-19.

[18] E. E. Akortha and O. K. Ibadin, "Incidence and Antibiotic Susceptibility Pattern of Staphylococcus Aureus amongist Patients with Urinary Tract Infection(UTI) in UBTH Benin City, Nigeria," African Journal of Biotechnolology, Vol. 7, No. 11, 2008, pp. 1637-1640.

[19] T. M. Hooton, "Fluoroquinolones and Resistance in the Treatment of Uncomplicated Urinary Tract Infection. International Journal of American Medicine Association," Vol. 22, No. 1, 2003, pp. S65-S72.

[20] P. Nwanze, L. M. Nwara, S. Oranusi, et al., "Urinary Tract Infection in Okada Village: Prevalence and Antimicrobial Pattern," Science of Research Essays, Vol. 2, No. 4, 2007, pp. 112-116.

[21] O. A. H. Al-Jebouri, "The Relationship between Urinary Caliculi Types and Urinary Tract Infections among Patients in Tikrit District," M. Sc. Thesis, University of Tikrit, Tikrit, 2006. 